\title{
Culturally Responsive Social Robotics Instruction for Middle School Girls
}

\author{
Andrew B. Williams, Kathleen Baert, Adrianna Williams \\ Humanoid Engineering and Intelligent Robotics Lab \\ Marquette University \\ andrew.williams@marquette.edu
}

\begin{abstract}
To attract more female students to study human-robot interaction (HRI) and robotics in college, new approaches for engaging middle and high school students need to be developed. This paper presents a unique approach to teaching middle school girls primary human-robot interaction concepts and humanoid robot programming using culturally responsive pedagogy. We describe what culturally responsive social robotics (CRSR) instruction is, how we incorporated CRSR and human-robot interaction instruction into a middle school girls social robotics program, and how we evaluated its effectiveness using mixed methods evaluation.
\end{abstract}

\section{Keywords}

social robotics; culturally responsive robotics; education

\section{INTRODUCTION}

In this paper, we present a new culturally responsive approach to teaching middle school and high school girls concepts in human-robot interaction and how to program humanoid robots with limited social capabilities. Social robotics is the study of how co-robots, or collaborative robots, can be developed to cooperate with humans through intelligent conversational, emotional, and physical interaction $[1,2]$. Social robots are contrasted with traditional robotics by their ability to speak, emote, and learn and by how their users tend to anthropomorphize them. This project seeks to increase the participation of girls in social robotics by developing a culturally responsive social robotics curriculum for middle school girls.

Our program teaches middle school girls primary humanrobot interaction (HRI) concepts including humanoid robot programming, robot ethics, and STEM concepts integrated with the culturally responsive aspects of asset building, reflection, and connectedness to their communities. Culturally responsive social robotics pedagogy incorporates 1) asset building, 2) reflection, and 3) connectedness as ways to improve instructional effectiveness, particularly with students

Permission to make digital or hard copies of part or all of this work for personal or classroom use is granted without fee provided that copies are not made or distributed for profit or commercial advantage and that copies bear this notice and the full citation on the first page. Copyrights for third-party components of this work must be honored. For all other uses, contact the owner/author(s).

HRI '17 Companion March 06-09, 2017, Vienna, Austria

(C) 2017 Copyright held by the owner/author(s).

ACM ISBN 978-1-4503-4885-0/17/03.

DOI: http://dx.doi.org/10.1145/3029798.3038427

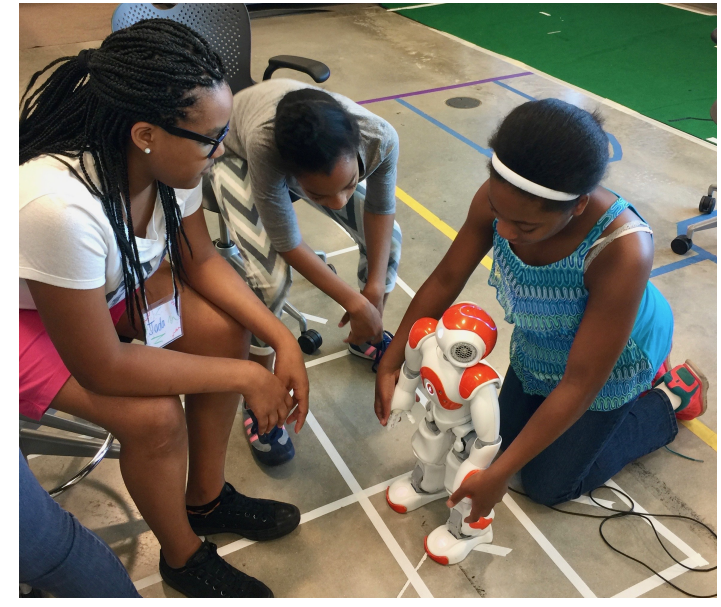

Figure 1: Middle school girls learning to program the Nao social robot.

under-represented in the sciences and engineering, such as women and minorities [3]. Asset building, as opposed to deficit-based instruction, encourages teachers and students to focus on aspects of their identity that can be seen as strengths. With asset building, social robotics students can be more engaged in the workshop instruction and lectures when the instructors recognize the value of students' knowledge and create a bridge to the robot programming skills that need to be learned. With reflection, social robotics students are taught to ponder the knowledge they learn and challenge themselves to think more deeply about how it relates to their topics of interest, identities, and behaviors. The culturally responsive aspect of connectedness means that students feel connected to each other and their communities when they are immersed in a peer culture that fosters a sense of responsibility to something greater than themselves.

\section{APPROACH}

In this section, we summarize the topics and activities that are covered in our culturally responsive social robotics fiveday workshop. Our approach involves incorporating culturally responsive pedagogy and social justice topics with social robotics programming in a one-week middle school workshop for girls and measuring the impact through qualitative and quantitative evaluation. The CRSR curriculum we developed includes a student instructional workbook as well as 
teacher training materials. In this section, we describe the topics covered in the five days to demonstrate how we integrate culturally responsive pedagogy with instruction in social robotics and programming. It is important to note that the topics and curriculum are designed to teach to students who may have no prior robotics knowledge or interest.

On the first day, the CRSR curriculum introduces STEM concepts, programming and debugging robots, robot sensors and motors, and robot handling and safety in the context of culturally responsive social robotics aspects of asset building, reflection, and connectedness. Although our workshop used Nao humanoid robots, the curriculum can be adapted to use with other social robots. The students are asked to design and draw their perfect robot, describe what it would do and discuss its significance. The students reflect on their identity by creating an "I Am" poem. We also teach the students how to program the social robot using the visual robot programming environment, Choreographe. We teach them how to program through presentations on the projector, live demonstrations on the projector, and working one on one. The students are then shown how to create human-robot dialogues using Choreographe.

Day 2: Subsequent days involve teaching a variety of human-robot interaction and social robot programming activities that incorporate culturally responsive pedagogy. The HRI and social robotics topics include using the Choice and Dialog programming constructs, creating custom motion animations, and incorporating music for a robot dance contest. Day 3: Ballet is introduced to demonstrate perceptions of movement and gender and is used to relate it to robot motions. Students reflect on and discuss robot ethics using the 2016 Scientific American article, "Why robots need to be able to say no," as a basis for the discussion. Day 4: Other culturally responsive activities include the study of emotions in humans and robots, and the gender inequities in technology, including emoji design. Slam poetry is used for a learning activity involving female STEM role models in the context of a discussion of intersectionality. The final day is the presentation of a week-long student research project that includes a social robot demo that shows how they used a social robot to address, solve, or raise awareness for a social justice issue they researched. An example of a research project the students completed included studying gender inequity in STEM careers and a demo of social robot avatars used for a job interviewer and interviewee to prevent unconscious gender bias.

\section{EVALUATION}

Our workshop was evaluated with U.S. minority populations that included middle school Latina and African American girls. Figures 2 and 3 show data from our four-week workshop. For our one-week workshop, descriptive statistics were also used to describe students' responses to two questionnaires. An analysis of data revealed a statistically significant difference between pre- and post-survey mean responses for aggregate items on each instrument. Findings suggest that because of our culturally responsive social robotics program, participants had increases in their understanding about the relationship between technology and social justice and had increases in their self-concepts related to computer science and technology. Findings also revealed statistically significant increases in computer self-efficacy. A descriptive analysis was performed on the post-survey re-

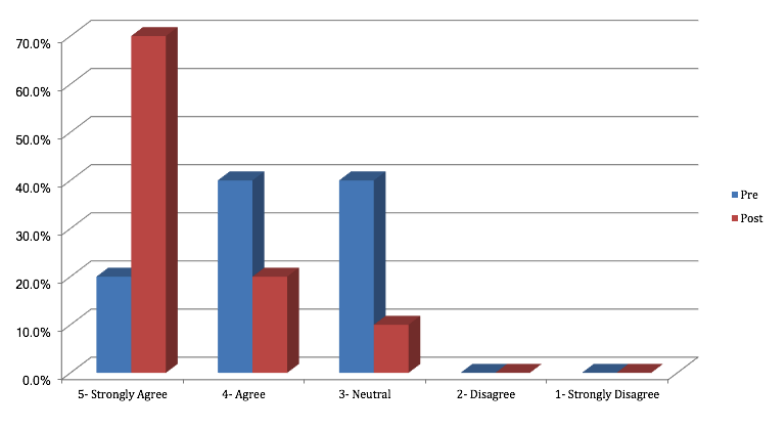

Figure 2: Whatever I learn about computer programming and robotics will be important for my future occupational success.

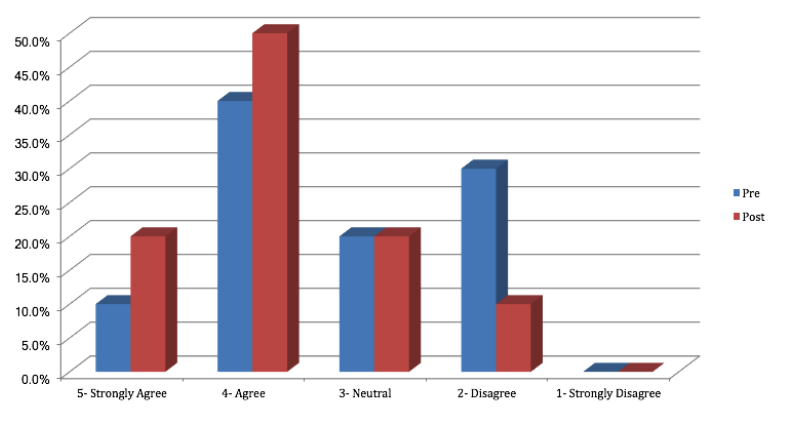

Figure 3: I can see myself programming robots after high school

sponses to understand girls' perceptions of their overall summer experience. Eight of 10 items had mean scores above 4 on a 5 point Likert scale. Participants rated the overall camp 4.16 on a 5 point scale. This rating indicates an excellent to very good summer camp experience for the participants.

\section{CONCLUSION}

Our culturally responsive social robotics curriculum and workshop approach has shown promising results in engaging and educating middle school girls in social robotics.

\section{ACKNOWLEDGMENTS}

This work was supported by the U.S. National Science Foundation grant IIS-1427399. The authors would like to thank and acknowledge the contributions of Kimberly Scott, Angelicque Blackmon, and others to this work.

\section{REFERENCES}

[1] C. L. Breazeal. Designing sociable robots. MIT press, 2004.

[2] E. Russell and A. B. Williams. Effects of SMILE emotional model on humanoid robot user interaction. In Proceedings of the Tenth Annual ACM/IEEE International Conference on Human-Robot Interaction, pages 113-114. ACM, 2015.

[3] K. A. Scott, K. M. Sheridan, and K. Clark. Culturally responsive computing: a theory revisited. Learning, Media and Technology, 40(4):412-436, 2015. 